\title{
TESIS DOCTORALES ESPAÑOLAS EN EDUCACIÓN MATEMÁTICA*
}

\author{
TORRALBO, MANUEL ${ }^{1}$, FERNÁNDEZ CANO, ANTONIO ${ }^{2}$, RICO, LUIS $^{3}$, MAZ, ALEXANDER $^{1}$ \\ Y GUTIÉRREZ, M. DEL PILAR ${ }^{1}$ \\ ${ }_{1}^{1}$ Departamento de Matemáticas. Universidad de Córdoba \\ ${ }^{2}$ Departamento de MIDE. Universidad de Granada \\ ${ }^{3}$ Departamento de Didáctica de la Matemática. Universidad de Granada
}

\begin{abstract}
Resumen. En este articulo se informa de una investigación sobre la producción de tesis doctorales de educación matemática en las universidades españolas desde 1976 a 1998. Se realizó un estudio cientimétrico y conceptual en ellas, detectándose patrones, tendencias y tópicos de gran actividad investigadora. Finalmente es constatada la consolidación del área de didáctica de las matemáticas como disciplina científica en el ámbito de la investigación española.

Palabras clave. Tesis doctorales, cienciometría, investigación, educación matemática.

Summary. This paper reports a research on the production of doctoral theses ( $\mathrm{Ph}, \mathrm{D}$, ) on Maths education in Spanish Universities from 1976 to 1998. A scientimetric and conceptual study was carried out in order to detect standards, trends and topic areas with a high research activity. Finally, the study confirms the consolidation of the area of Maths education as a scientific discipline in the Spanish research context.

Keywords. Doctoral dissertations, scientimetric, investigation, math education.
\end{abstract}

\section{INTRODUCCIÓN}

La educación matemática como campo de investigación es aún joven; sin embargo, es fuente de muchos estudios con métodos y paradigmas variados; este aspecto es consecuencia de que recibe aportaciones de diversas áreas como la psicología, pedagogía, filosofía, matemáticas e historia de las ciencias, entre otras. Tal variedad de contribuciones hace que afloren distintas facetas y consideraciones dinámicas entre la teoría y la práctica en educación matemática (Torralbo et al., 2001); así mismo hay enriquecimiento con las interacciones que se establecen en los procesos de enseñanza-aprendizaje de las matemáticas como consecuencia de esta múltiple conexión de la educación matemática.
Pese a estos matices, la investigación en educación matemática tiene dos propósitos principales (Shoenfeld, 2000; p. 41): uno puro a fin de entender la naturaleza del pensamiento matemático, la enseñanza y el aprendizaje y otro aplicado a fin de usar tales comprensiones para mejorar la instrucción de las matemáticas. Estos propósitos están enmarcados dentro de la perspectiva compartida por la comunidad de investigadores españoles del área, sobre la educación matemática como un conjunto de ideas, conocimientos, procesos, actitudes y, en general, de actividades implicadas en la construcción, representación, transmisión y valoración del conocimiento matemático que tiene lugar con carácter intencional (Rico y Sierra, 2000, p. 79). 
La educación matemática ha sido desarrollada como una disciplina académica estudiada en las universidades (Kilpatrick,1994), pero la cuestión primordial es «¿Qué es investigar en educación matemática?» (Gjone, 1998) y, particularmente en los estudios de doctorado, «¿Qué se investiga?». De esta manera, las mismas tesis doctorales deben ser objeto de análisis y reflexión con el propósito de indagar qué tópicos son los que atraen la atención de la comunidad académica a través de los estudios de tercer ciclo en educación matemática. Esto también permite detectar en qué medida están cambiando, tanto la visión como los paradigmas de la investigación en el área (Gravemeijer, 1994), así como identificar qué conceptos matemáticos se investigan más y cuáles se han marginado.

Conocer el estado de la investigación y sus resultados en los años recientes en educación matemática por medio de las tesis doctorales permite plantear propuestas de innovación en estrategias y procesos de enseñanza y aprendizaje, así como difundir los nuevos métodos de investigación utilizados. Conocer estos aspectos tiene importancia porque el determinar los patrones de productividad investigadora permite otorgar estatus científico a una disciplina mediante la similitud o la asimilación de éstos a los de disciplinas plenamente consolidadas (Fernández Cano y Bueno, 1999).

La variedad de aportaciones al área se refleja en el hecho de que incluso tesis doctorales en educación matemática realizadas en diferentes departamentos de una misma facultad o universidad pueden variar ampliamente en aspectos conceptuales, metodológicos y de paradigma (Gjone, 1998). Pese a esto, la tesis doctoral es la parte más importante de la formación académica del investigador.

Estos mencionados aspectos de las tesis doctorales en educación matemática no han sido muy explorados, quizá sea porque, como indican Duffin y Simpson (2000), la validación metodológica la otorgan sus vecinos metodológicos, es decir, los examinadores de la tesis y no la comunidad científica a través de publicaciones o conferencias.

En la investigación con tesis de ámbito internacional, Fiorentini (1993) abordó el problema de la divulgación, dispersión y discontinuidad de las investigaciones en educación matemática en Brasil, analizando 190 tesis de maestría, 12 tesis doctorales y 2 trabajos para obtener la titulación de libre-docencia, y centrándose sobre todo en las tendencias temáticas y teórico-metodológicas, de las indagaciones que forman el objeto de tales investigaciones. También Kilpatrick (1994) realizó un estudio sobre la historia de la investigación en educación matemática. Por medio de un grafico, presenta los datos de las tesis y de las disertaciones en educación matemática, mayoritariamente de Norteamérica, recopilados por Suydam, y los compara con los datos bibliográficos de Frosbissher y Joy sobre lo realizado en Inglaterra e Irlanda.

Reys (2000) estudió las tesis doctorales norteamericanas en educación matemática desde 1980 hasta 1998, incidiendo en los aspectos cientimétricos. Se desprende de ese estudio que el número de tesis doctorales ha permanecido estable en esos veinte años, con una media anual de 70 tesis y con una producción total de 1.386 (Reys, 2000). Analiza algunos factores que provocan una escasez de doctores en educación matemática pese a la demanda de profesionales en el área; así mismo, el estudio revela que menos de cincuenta instituciones producen un $85 \%$ de las tesis doctorales en dicha área.

En España, Torralbo y otros (2001) realizaron un estudio conectando tesis doctorales en educación matemática realizadas en España con el aumento y cualificación del cuerpo de docentes del área de didáctica de la matemática en las universidades españolas. Posteriormente, Torralbo (2001) realizó un estudio cientimétrico, conceptual y metodológico de las tesis doctorales españolas realizadas en educación matemática desde 1976 hasta 1998, identificando ciertos patrones y tendencias; tambien aporta una base de datos específica de tales tesis.

En este artículo presentamos una visión de aspectos conceptuales que se investigan en las tesis doctorales en un lapso de veintidós años, así como la productividad y algunas características cientimétricas en educación matemática. De entre las leyes básicas de la cienciometría que se aplican a este tipo de estudios, mencionaremos la ley de la dispersión de la literatura científica de Bradford, la cual dice que, si se consulta literatura especializada sobre un determinado tema, éste será publicado en un pequeño número de revistas (núcleo) y a partir de esta zona, si queremos recuperar el mismo número de artículos, hará falta un número muy superior de revistas y así sucesivamente, siendo el número de revistas en el núcleo y en las zonas siguientes como $1, n, n 2 \ldots$. (Turuget, 1989); para este trabajo hemos aplicado a ley de Bradford a la producción institucional de tesis doctorales.

\section{METODOLOGÍA}

La didáctica de la matemática es una disciplina emergente en España con pocos años de vida académica. Su incorporación a la universidad es posterior a la mayoría de las restantes disciplinas matemáticas, lo cual se logra a partir de 1984 con la promulgación de la Ley de Reforma Universitaria (LRU) (Rico, 1999a); por tanto, cuenta con una corta tradición investigadora. Tal vez por este motivo ocurre que muchos artículos de investigación en las revistas específicas de educación matemática en España se nutren de las tesis doctorales (Torralbo, 2001).

Este trabajo pretende ser una revisión integrada de un total de 135 tesis doctorales españolas de educación matemática, catalogadas en un período de tiempo establecido entre 1976 y 1998, con el fin de construir una visión general y sistemática de cómo se ha venido abordando la investigación en el ámbito de la educación matemática en lo conceptual, así como identificar aspectos cientimétricos que la caracterizan. Se pretendió cubrir todas las tesis desarrolladas en España que tuvieran relación con el campo en cuestión. La recogida de información de diversa índole y procedencia, su análisis e interpretación como elementos fundamentales en el trabajo de campo llevó a un examen 
crítico de los patrones generales que emergen de las tesis.

El estudio puede concretarse en las siguientes cuestiones: ¿Cuáles son los indicadores conceptuales que caracterizan las tesis doctorales españolas en educación matemática? ¿Pueden identificarse indicadores cientimétricos que informen sobre el estado de la investigación española en el área, referidos a tesis doctorales?

Se pretende caracterizar un aspecto fundamental del contenido de las tesis doctorales: diferenciar temas de investigación en educación matemática y crear un panorama general de aplicación.

\section{Población y muestra}

La población objeto de este estudio la forman todas las tesis doctorales españolas en educación matemática leídas en universidades españolas en un período comprendido entre 1976 y 1998. Se analizó si el objeto de estudio de tales tesis tiene relación con la enseñanza y el aprendizaje de las matemáticas en cualquiera de los niveles educativos, con la innovación, el estudio y el desarrollo curricular, la formación del profesorado de matemáticas o la fundamentación teórica del área de conocimiento de didáctica de la matemática, para establecer si correspondían o no a la población elegida.

El sistema que se ha seguido para hallar las tesis sobre educación matemática, que integran el estudio, ha sido como sigue:

- Búsqueda informática en la base de datos TESEO.

- Consultas al Centro de Documentación Thales y a las revistas Enseñanza de las Ciencias, Revista de Investigación Educativa, Epsilon, SUMA y a los Boletines de la Sociedad Española de Investigación en Educación Matemática.

- Mediante citaciones cruzadas, teniendo en cuenta las siguientes actuaciones:

Se ha recogido una lista del profesorado universitario en servicio activo, clasificados por áreas de conocimiento. Estas áreas son: didáctica de la matemática, psicología evolutiva y de la educación, didáctica y organización escolar y métodos de investigación y diagnóstico en educación. La lista se obtuvo de la Secretaría General del Consejo de Universidades, con fecha del 26 de abril de 1999.

\section{Recogida de datos}

Se utilizó un instrumento de recogida de datos estandarizado obtenido del ZDM (Zentralblatt für Didaktik der Mathematik), validado por el uso generalizado en la comunidad científica de educadores matemáticos europeos, el cual se compone de catorce variables. El esquema de clasificación de ZDM se organiza de la siguiente forma: todas las categorías temáticas están representadas por una anotación de tres dígitos consistente en una letra seguida de dos dígitos. La letra mayúscula determina las distintas clases tales como álgebra, geometría o material de enseñanza y multimedia instruccional. El primer dígito subdivide las clases en subclases especiales. El segundo dígito permite la caracterización del campo educativo en educación primaria, secundaria, etc.

Para la parte cientimétrica se elaboró un instrumento de 35 variables que fundamentalmente tiene un carácter descriptivo, el cual fue sometido a consulta de expertos en el área para constatar su fiabilidad.

Tabla I

Productividad diacrónica.

\begin{tabular}{|c|c|c|}
\hline Año de lectura & Frecuencia & $\%$ \\
\hline 1976 & 1 & $0,7 \%$ \\
\hline 1977 & 0 & $0 \%$ \\
\hline 1978 & 1 & $0,7 \%$ \\
\hline 1979 & 1 & $0,7 \%$ \\
\hline 1980 & 0 & $0 \%$ \\
\hline 1981 & 1 & $0,7 \%$ \\
\hline 1982 & 1 & $0,7 \%$ \\
\hline 1983 & 1 & $0,7 \%$ \\
\hline 1984 & 0 & $0 \%$ \\
\hline 1985 & 2 & $1,5 \%$ \\
\hline 1986 & 5 & $3,7 \%$ \\
\hline 1987 & 4 & $3 \%$ \\
\hline 1988 & 7 & $5,2 \%$ \\
\hline 1989 & 6 & $4,4 \%$ \\
\hline 1990 & 4 & $3 \%$ \\
\hline 1991 & 13 & $9,6 \%$ \\
\hline 1992 & 8 & $6 \%$ \\
\hline 1993 & 8 & $6 \%$ \\
\hline 1994 & 15 & $11,1 \%$ \\
\hline 1995 & 15 & $11,1 \%$ \\
\hline 1996 & 14 & $10,4 \%$ \\
\hline 1997 & 17 & $12,6 \%$ \\
\hline 1998 & 11 & $8,1 \%$ \\
\hline Total & 135 & \\
\hline
\end{tabular}


Figura1

Productividad diacrónica por cuatrienios.

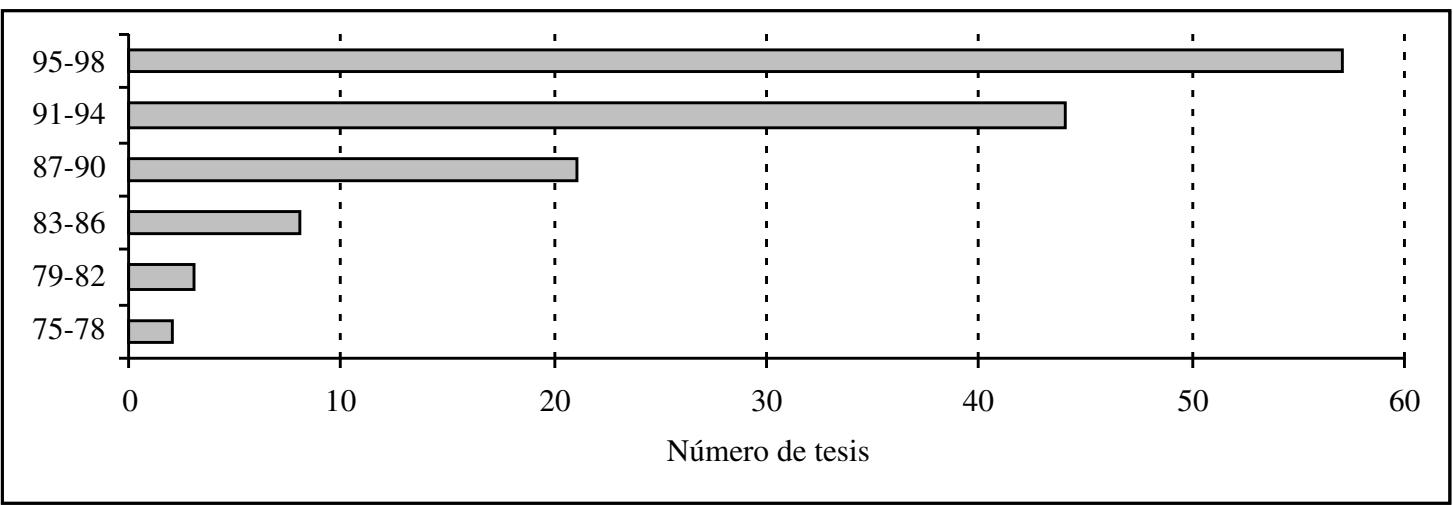

\section{RESULTADOS}

Vamos a presentar algunos de los resultados significativos que ha revelado el estudio. Para ello se han agrupado en dos bloques, uno de aspectos cientimétricos y otro de conceptuales.

\section{Aspectos cientimétricos}

\section{- Productividad diacrónica}

La productividad diacrónica de la educación matemática en el período 1976-98 (Tabla I) crece de manera paulatina a partir del año 1985; aunque no será hasta los años noventa (específicamente en el año 1991) cuando se origina un crecimiento más acentuado, crecimiento que se prolonga actualmente sin alcanzar todavía un período de madurez propio de una disciplina ya asentada en el campo de la investigación. En la figura 1 se puede apreciar la evolución de la productividad, agrupada por cuatrienios.

El incremento en la producción de tesis doctorales en educación matemática no sólo han impulsado la consolidación de los programas de doctorado en el área de didáctica de la matemática, sino también, como afirma Rico (1999b), la formación de un nuevo profesorado universitario motivado e interesado en la investigación.

\section{- Instituciones}

A partir de la tabla II, agrupamos la productividad institucional de las tesis doctorales en las universidades españolas en tres bloques: grandes productoras de tesis, instituciones de producción media e instituciones ocasionales en la producción de tesis. Éstas son:

- Grandes productoras de tesis: Universidades de Granada, Complutense de Madrid, Autónoma de Barcelona, Barcelona, Valencia y Nacional de Educación a Distancia (UNED).
Tabla II

Productividad institucional.

\begin{tabular}{|c|c|}
\hline Instituciones de lectura & Frecuencia \\
\hline U. Granada & 22 \\
\hline U. Complutense de Madrid & 18 \\
\hline U. Autónoma de Barcelona & 14 \\
\hline U. Barcelona & 14 \\
\hline U. Valencia & 12 \\
\hline UNED & 11 \\
\hline U. Murcia & 6 \\
\hline U. Sevilla & 6 \\
\hline U. País Vasco & 5 \\
\hline U. La Laguna & 5 \\
\hline U. Cádiz & 5 \\
\hline U. Politécnica de Madrid & 3 \\
\hline U. Autónoma de Madrid & 2 \\
\hline U. Oviedo & 2 \\
\hline U. Salamanca & 2 \\
\hline U. Santiago & 2 \\
\hline U. Valladolid & 2 \\
\hline U. Zaragoza & 1 \\
\hline U. Huelva & 1 \\
\hline U. Vigo & 1 \\
\hline U. Politécnica de Valencia & 1 \\
\hline Total & 135 \\
\hline
\end{tabular}


Figura 2

Zonas Bradford de productividad institucional.

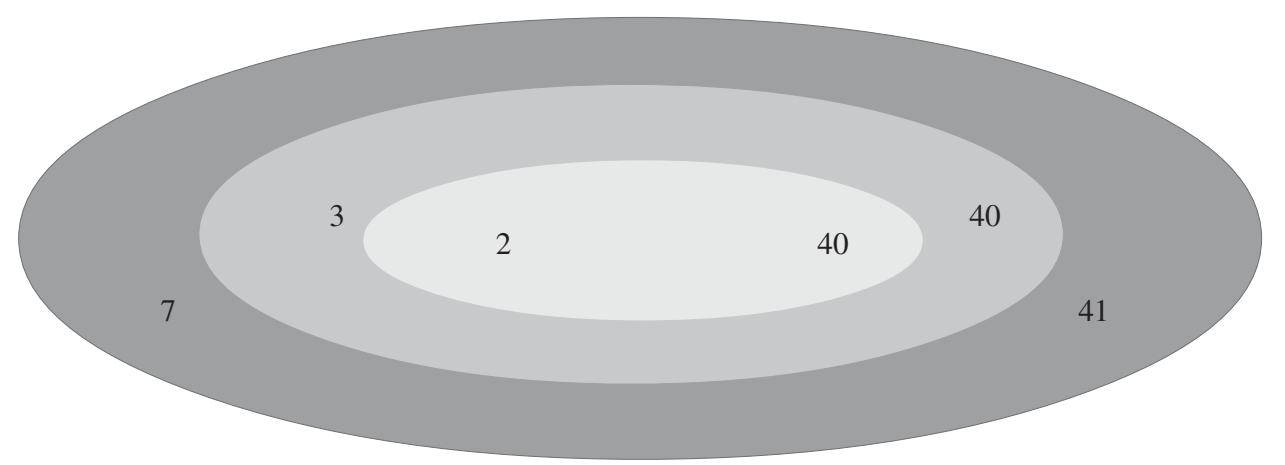

- Instituciones de producción media: Universidades de Oviedo, Sevilla, País Vasco, La Laguna, Cádiz, Politécnica de Madrid, Autónoma de Madrid, Salamanca, Santiago y Valladolid.

- Instituciones ocasionales en la producción de tesis: Universidades de Zaragoza, Huelva y Politécnica de Valencia.

Es de destacar que en la Universidad de Granada se han realizado el 15,6\% y en la Complutense de Madrid, el $13,3 \%$, sumando entre ambas más de la cuarta parte del total de tesis doctorales culminadas en España en el período 1976-98.

Aplicando la ley de Bradford se puede clarificar la producción de las universidades españoles, en este caso el núcleo está constituido por las universidades de Granada y la Complutense de Madrid, con una producción conjunta de 40 tesis doctorales. En una primera zona y con la misma producción que el núcleo están la Universidad Autónoma de Barcelona, la Universidad de Barcelona y la Universidad de Valencia. En una segunda zona están la UNED, la Universidad de Murcia, la Universidad de Sevilla, la Universidad del País Vasco, la Universidad de La Laguna, la Universidad de Cádiz y la Universidad Politécnica de Madrid, que entre ellas reúnen una producción de 41 tesis. (Figura 2)

\section{Núcleo}

Universidad de Granada

Universidad Complutense de Madrid

\section{Primera zona}

Universidad Autónoma de Barcelona

Universidad de Barcelona

Universidad de Valencia

\section{Segunda zona}

UNED

Universidad de Murcia

Universidad de Sevilla

Universidad del País Vasco

Universidad de La Laguna

Universidad de Cádiz

Universidad Politécnica de Madrid.

\section{Periferia}

En la periferia se encontrarían las 14 universidades restantes.

\section{- Departamentos}

Los datos presentados en la tabla III, indican cuáles son las áreas de conocimiento más fructíferas en educación matemática.

\section{Tabla III}

Departamento de realización de la tesis doctoral.

\begin{tabular}{|l|c|c|}
\hline Departamentos de realización & Frecuencia & $\%$ \\
\hline Pedagógicos & 53 & $39,2 \%$ \\
\hline Didácticas específicas & 40 & $29,6 \%$ \\
\hline Psicológicos & 24 & $17,8 \%$ \\
\hline Matemáticos & 11 & $8,1 \%$ \\
\hline Otros & 7 & $5,2 \%$ \\
\hline Total & $\mathbf{1 3 5}$ & \multicolumn{1}{|l}{} \\
\hline
\end{tabular}


En la productividad de tesis en educación matemática destacan, en primera instancia, los departamentos relacionados con la educación pedagógica, con un $39,2 \%$; en segundo lugar, los departamentos específicos, como didáctica de la matemática y didáctica de las ciencias experimentales, con un 29,6\%. Hacemos referencia especial al hecho de que la productividad de los departamentos de didáctica de la matemática resulta significativa a partir del año 1993. (Figura 3)

Las tesis doctorales producidas desde los departamentos de psicología han sido 24 y representan el 17,8\%, Este dato es bastante paradójico, puesto que, cuando lo relacionamos con el análisis de datos conceptuales, se observa que la psicología representa un campo de estudio muy considerado en este tipo de investigaciones.

\section{- Directores}

Los directores de tesis doctorales en educación matemática más productivos en estos 22 años aparecen relacionados en la tabla IV.

En total, 115 directores han dirigido o codirigido estas tesis entre 1976 y 1998, y tan sólo 5 de ellos dirigieron el $19,25 \%$ del total. También es destacable que la mayoría de estos directores pertenecen al área de didáctica de la matemática.
Tabla IV

Relación de los directores más productivos.

\begin{tabular}{|c|c|c|}
\hline Directores & Frecuencia & $\%$ \\
\hline Rico Romero, Luis & 7 & $15,9 \%$ \\
\hline Batanero Bernabeu, M. Carmen & 6 & $13,6 \%$ \\
\hline Gutiérrez Rodríguez, Ángel & 5 & $11,4 \%$ \\
\hline De la Orden Hoz, Arturo & 4 & $9,1 \%$ \\
\hline Díaz Godino, Juan & 4 & $9,1 \%$ \\
\hline Serrano González, M.J. & 3 & $6,8 \%$ \\
\hline García Hoz, Víctor & 3 & $6,8 \%$ \\
\hline Fortuny Aymemi, José M. & 3 & $6,8 \%$ \\
\hline Navarro Guzmán, José I. & 3 & $6,8 \%$ \\
\hline Villar Angulo, Luis Miguel & 3 & $6,8 \%$ \\
\hline Fernández Arenaz, A. & 3 & $6,8 \%$ \\
\hline Johnson, David & 3 & $6,8 \%$ \\
\hline Total & 47 & \\
\hline
\end{tabular}

Departamentos de realización en relación con el año de lectura en cuatrienios.

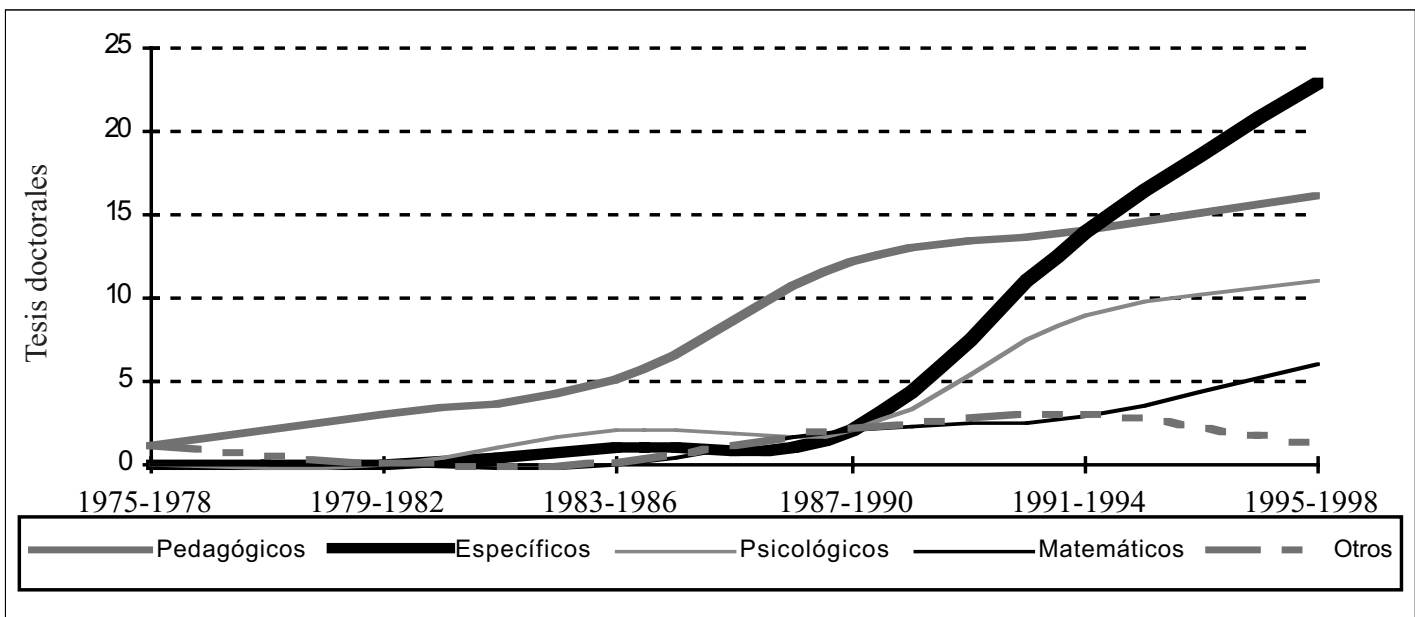

- Revistas más citadas

El estudio revela que las revistas más citadas, a cierta distancia de las demás, son Educational Studies in
Mathematics y Journal for Research in Mathematics Education en un total de 57 y 54 tesis respectivamente. Se destacan 7 revistas propias del ámbito de la educación matemática y 3 de la psicología; además es importante la 
Tabla V

Revistas más citadas.

\begin{tabular}{|l|l|c|}
\hline Rango & \multicolumn{1}{|c|}{ Revistas más citadas } & Frecuencias \\
\hline $1^{\circ}$ & Educational Studies in Mathematics & 57 \\
\hline $2^{\circ}$ & $\begin{array}{l}\text { Journal for Research in Mathematics } \\
\text { Education }\end{array}$ & 54 \\
\hline $3^{\circ}$ & $\begin{array}{l}\text { Recherches en Didactique des } \\
\text { Mathématiques }\end{array}$ & 28 \\
\hline $4^{\circ}$ & Journal of Educational Psychology & 18 \\
\hline $5^{\circ}$ & For the Learning of Mathematics & 17 \\
\hline $6^{\circ}$ & Enseñanza de las Ciencias & 15 \\
\hline $7^{\circ}$ & Infancia y Aprendizaje & 15 \\
\hline $8^{\circ}$ & Mathematics Teacher & 14 \\
\hline $9^{\circ}$ & Arithmetic Teacher & $\mathbf{2 4 6}$ \\
\hline $10^{\circ}$ & Cuadernos de Pedagogía & 13 \\
\hline & Total & 15 \\
\hline
\end{tabular}

Tabla VI

Relación de los autores más citados.

\begin{tabular}{|l|l|c|}
\hline Rango & \multicolumn{1}{|c|}{ Autores más citados } & Frecuencia \\
\hline $1^{\circ}$ & Piaget, Jean & 41 \\
\hline $2^{\circ}$ & Carpenter, Thomas & 18 \\
\hline $3^{\circ}$ & Coll, César & 16 \\
\hline $4^{\circ}$ & Rico, Luis & 15 \\
\hline $5^{\circ}$ & Schoenfeld, Alan & 15 \\
\hline $6^{\circ}$ & Vergnaud, Gerard & 13 \\
\hline $7^{\circ}$ & Bell, Alan & 11 \\
\hline $8^{\circ}$ & Brousseau, Guy & 10 \\
\hline $9^{\circ}$ & Fischbein, Efraim & 9 \\
\hline $10^{\circ}$ & Díaz Godino, Juan & $\mathbf{1 5 9}$ \\
\hline & Total & \\
\hline & & 11 \\
\hline
\end{tabular}

presencia de tres revistas españolas entre las más citadas, lo cual indica un interés por utilizar las producciones e investigaciones del país. (Tabla V)
Tabla VII

Frecuencia de tesis doctorales en relación con las variables conceptuales.

\begin{tabular}{|c|c|c|}
\hline Variables & Frecuencia & $\%$ \\
\hline A: General & 11 & $8,1 \%$ \\
\hline B: Política educativa y sistema educativo & 20 & $14,8 \%$ \\
\hline $\begin{array}{l}\text { C: Psicología de la educación matemática. } \\
\text { Investigación en educación matemática. } \\
\text { Aspectos sociales }\end{array}$ & 109 & $80,7 \%$ \\
\hline D: Educación e instrucción en matemáticas & 112 & $83 \%$ \\
\hline E: Fundamentos de las matemáticas & 15 & $11,1 \%$ \\
\hline $\begin{array}{l}\text { F: Aritmética. Teoría de los números } \\
\text { Cantidades. }\end{array}$ & 22 & $16,3 \%$ \\
\hline G: Geometría & 22 & $16,3 \%$ \\
\hline H: Álgebra & 7 & $5,2 \%$ \\
\hline I: Análisis & 10 & $7,4 \%$ \\
\hline $\begin{array}{l}\text { K: Combinatoria y teoría de grafos. } \\
\text { Estadística y probabilidad }\end{array}$ & 10 & $7,4 \%$ \\
\hline $\begin{array}{l}\text { M: Modelos matemáticos. } \\
\text { Matemáticas aplicadas }\end{array}$ & 4 & $3 \%$ \\
\hline $\begin{array}{l}\text { N: Matemáticas numéricas. } \\
\text { Matemáticas discretas. } \\
\text { Software matemático }\end{array}$ & 5 & $3,7 \%$ \\
\hline U: Materiales y medios educativos & 25 & $18,5 \%$ \\
\hline Acumulado & 372 & \\
\hline
\end{tabular}

\section{- Autores}

Se pone de manifiesto cuáles son los autores más citados en las tesis doctorales, considerando la frecuencia dentro de una escala de los 10 más referenciados en cada tesis.

Aunque Piaget es claramente el autor mas citado, cabe destacar la presencia de tres autores españoles también entre los más citados: César Coll, Luis Rico y Juan Díaz Godino. (Tabla VI)

\section{Aspectos conceptuales}

El análisis de los datos conceptuales se realizó atendiendo a dos niveles: un nivel micro donde se analizaron algunas de las categorías temáticas pertenecientes a las variables del instrumento y un nivel macro en el que se examinaron de forma global las 14 variables del instrumento de recogida de datos. Por último, se realizó una matriz de contingencia para recoger la frecuencia de aparición de cada una de las variables respecto a las demás. Destacamos que algunas de las tesis doctorales pertenecen a dos o más categorías temáticas y, por tanto, a dos o más variables. Esta circunstancia hace que el valor de la frecuencia acumulada de algunas de las variables del instrumento no coincida 
con el número de tesis incluidas en las diferentes categorías temáticas.

\section{- Análisis global de datos conceptuales}

La investigación que se ha realizado en educación matemática entre 1976 y1998 en España a través de las tesis doctorales se orienta de manera general a dos grandes áreas de investigación:

En primer término, Educación e instrucción en matemáticas, que la polariza con un $83 \%$. Así tenemos que el mayor número de tesis son las que indagan sobre los procesos relacionados con la enseñanza y el aprendizaje desde la perspectiva de la educación. En segundo lugar, aparece la psicología de la educación matemática, investigación en educación matemática, aspectos sociales, la cual fue objeto de investigación en el $80,7 \%$ del total de tesis analizadas. La categoría temática de mayor presencia, tanto en esta variable como a nivel general, fue C30, Procesos cognitivos. Aprendizaje y teorías educativas, que se observa en 79 tesis; es la única categoría temática que es tratada

\section{Tabla VIII}

Frecuencias de tesis doctorales en relación con las categorías temáticas de la variable $\mathrm{D}$.

\begin{tabular}{|c|c|c|}
\hline Categorías temáticas & Frecuencia & $\%$ \\
\hline $\begin{array}{l}\text { D 10: Trabajos comprensivos y estudios } \\
\text { sobre la formación matemática general } \\
\text { y en diferentes niveles y tipos. } \\
\text { Estudios comparativos sobre la } \\
\text { educación matemática } \\
\text { en diferentes países. }\end{array}$ & 7 & $5,2 \%$ \\
\hline $\begin{array}{l}\text { D 20: Contribuciones teóricas y } \\
\text { filosóficas a la didáctica matemática. } \\
\text { Métodos de investigación. Teorías de la } \\
\text { educación de las matemáticas. }\end{array}$ & 6 & $4,4 \%$ \\
\hline $\begin{array}{l}\text { D 30: Objetivos de la enseñanza } \\
\text { de las matemáticas. Desarrollo } \\
\text { curricular (formación matemática. } \\
\text { Competencias mínimas. } \\
\text { Objetivos y contenidos). }\end{array}$ & 61 & $45,2 \%$ \\
\hline $\begin{array}{l}\text { D 40: Métodos de enseñanza y } \\
\text { técnicas de clase. Preparación } \\
\text { de la lección. } \\
\text { Principios educacionales. }\end{array}$ & 18 & $13,3 \%$ \\
\hline $\begin{array}{l}\text { D 50: Investigación y resolución } \\
\text { de problemas. }\end{array}$ & 36 & $26,7 \%$ \\
\hline D 60: Evaluación del alumnado. & 31 & $22,9 \%$ \\
\hline $\begin{array}{l}\text { D 70: Diagnóstico, análisis de dificultades } \\
\text { de aprendizaje, ideas equivocadas y } \\
\text { errores del estudiante. }\end{array}$ & 21 & $15,6 \%$ \\
\hline $\begin{array}{l}\text { D 80: Unidades de enseñanza, } \\
\text { documentación y temas de la lección. }\end{array}$ & 1 & $0,7 \%$ \\
\hline Acumulado & 181 & \\
\hline
\end{tabular}

Tabla IX

Frecuencias de tesis doctorales en relación con las categorías temáticas de la variable $\mathrm{C}$.

\begin{tabular}{|l|c|c|}
\hline Categorías temáticas & Frecuencia & $\%$ \\
\hline C 10: Trabajos de comprensión y estudios. & 1 & $0,7 \%$ \\
\hline C 20: Aspectos afectivos. & 5 & $3,7 \%$ \\
\hline $\begin{array}{l}\text { C 30: Procesos cognitivos. Aprendizaje, } \\
\text { teorías de aprendizaje. }\end{array}$ & 79 & $58,5 \%$ \\
\hline $\begin{array}{l}\text { C 40: Inteligencia y aptitudes. } \\
\text { Personalidad. }\end{array}$ & 7 & $5,2 \%$ \\
\hline \begin{tabular}{l} 
C 50: Lenguaje y comunicación. \\
\hline $\begin{array}{l}\text { C 60: Aspectos sociológicos del } \\
\text { aprendizaje. }\end{array}$
\end{tabular} & 7 & $5,2 \%$ \\
\hline $\begin{array}{l}\text { C 70: Procesos de enseñanza-aprendizaje. } \\
\text { Evaluación de la instrucción. }\end{array}$ & 29 & $21,5 \%$ \\
\hline $\begin{array}{l}\text { C 80: Otros aspectos psicológicos. } \\
\text { C 90: Otros aspectos educacionales } \\
\text { (educación especial, educacional } \\
\text { vocacional, andragogía). }\end{array}$ & 12 & $8,9 \%$ \\
\hline \begin{tabular}{l} 
Acumulado \\
\hline
\end{tabular} & $\mathbf{1 5 8}$ & $3,7 \%$ \\
\hline
\end{tabular}

en más del $50 \%$ de las tesis. Este resultado tiene su coherencia con el hecho de que el cognitivismo es la teoría predominante que fundamenta el mayor porcentaje de las tesis analizadas. (Tabla VII)

Veamos de forma detallada, por medio de las tablas VIII y IX, las dos categorías temáticas más estudiadas en las tesis españolas en educación matemática.

Atendiendo a las 135 tesis doctorales que forman parte de la muestra de estudio, 109 están incluidas en alguna de las categorías temáticas indicadas anteriormente (Tabla IX) y 26 no se incluyen en ninguna de estas categorías.

\section{- Contingencia de aspectos conceptuales}

A través de una tabla de contingencia se establecen las relaciones de las trece variables conceptuales entre sí, donde cada celda contiene el número de tesis que considera alguna categoría temática de las variables recogidas en el instrumento conceptual.

El análisis de la tabla de contingencia (Tabla X) pone de manifiesto que un alto número de tesis doctorales, concretamente un 67,4\%, abordan la investigación tanto en $E d u$ cación e instrucción en matemáticas como en Psicología de la educación matemática. Investigación en educación matemática. Aspectos sociales, de tal manera que esta com- 
Tabla X

Matriz de contingencia de variables incluidas en el estudio conceptual.

\begin{tabular}{|c|c|c|c|c|c|c|c|c|c|c|c|c|c|}
\hline & 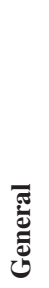 & 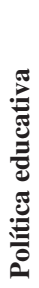 & $\begin{array}{l}\frac{\pi}{60} \\
\frac{0}{0} \\
\frac{0}{\mathscr{E}} \\
0\end{array}$ & 氖 & 莞 & 导 & 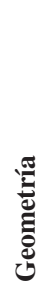 & $\frac{\pi}{\frac{0}{0}}$ & 厸 & 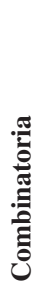 & 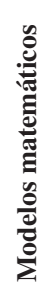 & 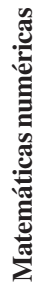 & 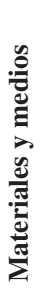 \\
\hline & $\mathbf{A}$ & B & C & D & $\mathbf{E}$ & $\mathbf{F}$ & G & $\mathbf{H}$ & I & $\mathbf{K}$ & $\mathbf{M}$ & $\mathbf{N}$ & $\mathbf{U}$ \\
\hline $\mathbf{A}$ & 11 & & & & & & & & & & & & \\
\hline B & 3 & 20 & & & & & & & & & & & \\
\hline $\mathbf{C}$ & 5 & 14 & 109 & & & & & & & & & & \\
\hline D & 10 & 17 & 91 & 112 & & & & & & & & & \\
\hline $\mathbf{E}$ & 1 & 1 & 11 & 11 & 15 & & & & & & & & \\
\hline $\mathbf{F}$ & 3 & 0 & 18 & 15 & 6 & 22 & & & & & & & \\
\hline $\mathbf{G}$ & 2 & 2 & 16 & 15 & 0 & 4 & 22 & & & & & & \\
\hline H & 0 & 0 & 3 & 7 & 2 & 0 & 0 & 7 & & & & & \\
\hline I & 1 & 3 & 6 & 9 & 2 & 0 & 0 & 1 & 10 & & & & \\
\hline $\mathbf{K}$ & 0 & 2 & 9 & 6 & 0 & 0 & 0 & 0 & 0 & 10 & & & \\
\hline $\mathbf{M}$ & 1 & 1 & 0 & 2 & 0 & 1 & 3 & 1 & 1 & 0 & 4 & & \\
\hline $\mathbf{N}$ & 0 & 0 & 4 & 4 & 0 & 0 & 1 & 0 & 0 & 1 & 0 & 5 & \\
\hline $\mathbf{U}$ & 4 & 1 & 18 & 20 & 3 & 2 & 8 & 1 & 2 & 1 & 1 & 2 & 25 \\
\hline
\end{tabular}

binación de áreas de investigación genera un nuevo campo de investigación. Los materiales y medios son el tercer campo temático en número de investigaciones realizadas en las tesis.

Asimismo la información obtenida nos lleva a establecer dos grandes categorías de investigación en educación matemática: zonas bastante indagadas y zonas poco indagadas.
Las zonas bastantes indagadas son las siguientes:

- Variable D: Educación e instrucción en matemáticas.

- Variable C: Psicología de la educación matemática. Investigación en educación matemática. Aspectos sociales.

- Variable C-D: Educación e instrucción en matemáticas, y psicología de la educación matemática. 
- Variable U: Materiales y medios educativos. Educación tecnológica.

- Variable F: Aritmética.

- Variable B: Política educativa y sistema educativo.

- Variable D-U: Educación e instrucción en matemáticas, y materiales y medios educativos.

Las zonas poco indagadas son las siguientes:

- Variable A-H: General y álgebra.

- Variable A-K: General, y combinatoria y teoría de grafos.

-Variable A-N: General y matemáticas numéricas.

- Variable B-F: Política educativa y aritmética.

- Variable B-H: Política educativa y álgebra.

- Variable B-N: Política educativa... y matemáticas numéricas.

- Variable C-M: Psicología de la educación matemática, y modelos matemáticos.

- Variable E-G: Fundamentos de las matemáticas y geometría.

- Variable E-K: Fundamentos de las matemáticas, y combinatoria y teoría de grafos.

- Variable E-M: Fundamentos de las matemáticas y modelos matemáticos.

- Variable E-N: Fundamentos de las matemáticas y matemáticas numéricas.

- Variable F-H: Aritmética y álgebra.

- Variable F-I: Aritmética y análisis.

- Variable F-K: Aritmética, y combinatoria y teoría de grafos.

- Variable G-H: Geometría y álgebra.

- Variable G-I: Geometría y análisis.

- Variable G-K: Geometría, y combinatoria y teoría de grafos.

- Variable H-K: Álgebra, y combinatoria y teoría de grafos.

- Variable I-N: Análisis y matemáticas numéricas.

- Variable K-M: Combinatoria y teoría de grafos, y modelos matemáticos.

- Variable M-N: Matemáticas numéricas, y materiales y medios educativos.
Hecho el análisis de las distintas variables, es pertinente comparar estas variables clasificatorias con la organización de los grupos de trabajos utilizada por la Sociedad Española de Investigación en Educación Matemática, SEIEM, por ser ésta el órgano que aglutina a los investigadores del área. Los grupos de investigación de la SEIEM son: Aprendizaje de la geometría, Conocimiento y desarrollo profesional del profesor, Didáctica del análisis, Didáctica de la estadística, probabilidad y combinatoria, Didáctica de la matemática como disciplina científica, Educación infantil, Historia de la educación matemática, Pensamiento numérico y algebraico (SEIEM, 2000).

De estos ocho grupos de investigación, tres (Aprendizaje de la geometría, Didáctica del análisis, Didáctica de la estadística, probabilidad y combinatoria) se ajustan a las variables clasificatorias del ZDM (G, I y K); un grupo de investigación (Pensamiento numérico y algebraico) agrupa a tres de las variables $(\mathrm{C}, \mathrm{F}$ y $\mathrm{H})$ y los cuatro restantes se ajustan a algunas de las subcategorías temáticas de las variables conceptuales consensuadas por el ZDM. Así mismo se evidencia que algunos grupos desarrollan su actividad de forma transversal a las variables clasificatorias utilizadas por el ZDM. Este análisis anterior pone de manifiesto cierto desajuste entre la caracterización de los grupos de investigación de la SEIEM y la producción doctoral detectada.

\section{CONCLUSIONES}

Este estudio, realizado a través del análisis conceptual de categorías temáticas, ha puesto de manifiesto que, pese a que en España la didáctica de la matemática como área de conocimiento es reciente, se ha investigado en sus diversos campos temáticos en la mayoría de las universidades españolas. Sin embargo, aparecen 39 de estas categorías temáticas que no han sido objeto de investigación, lo que nos reafirma en el hecho de que aún se encuentra en fase de crecimiento, sin llegar todavía al desarrollo propio de cualquier disciplina científica.

Es palpable el liderazgo de los departamentos de didáctica de la matemática y los de didácticas específicas que la incluyen, los cuales han empezado a asumir la investigación en educación matemática, desplazando a los departamentos de pedagogía. Esto es una clara evidencia de la importancia que ha tenido la implantación de los estudios de doctorado en didáctica de la matemática a partir de1988, tal como lo demuestran los resultados presentados.

La educación matemática en España ofrece aún campos de investigación totalmente vírgenes tal como se presenta en la tabla de contingencia, lo cual debe ser motivo de reflexión como de iniciativas por parte de los investigadores nacionales para indagar en dichos campos. Las zonas poco indagadas servirán de orientación para que los investigadores encaminen hacia estas sendas las nuevas tesis doctorales que se realicen en la universidad española.

El estudio permite comprobar que la producción investigadora en educación matemática de las tesis doctorales 
cumple con la ley de la dispersión de la literatura científica de Bradford, una de las leyes básicas de la cienciometría, lo cual es un claro indicio de que se está consolidando a nivel universitario en España.

Finalmente, hallamos que la presencia de investigadores españoles entre los autores más citados así como la aparición de revistas nacionales entre las más referenciadas es un indicador del esfuerzo y el progreso por parte de la comunidad académica española por incorporar, difundir y

\section{REFERENCIAS BIBLIOGRÁFICAS}

DUFFIN, J. y SIMPSON, A. (2000). When does a way of working become a methodology? Journal of Mathematical Behavior, 19 , pp. $175-188$.

FERNÁNDEZ CANO, A. y BUENO A. (1999). Synthesizing scientometric haterous in Spanish educational research. Scientometrics, 46(2), pp. 349-367.

FIORENTINI, D. (1993). Memoria e análisis da pesquisa académica em educaçao matemática no Brasil: $\mathrm{O}$ banco de teses do CEMPEM/FE-UNICAM. Revista Zetetiké, 1(1), pp. 55-76.

GJONE, G. (1998). Programs for the education of researchers in mathematics education, en Sierpinska, A. y Kilpatrick, J. (eds.). Mathematics education as a research domain: a search for identity, pp. 177-195. Gran Bretaña: Kluwer.

GRAVEMEIJER, K. (1994). Educational development and developmental research in mathematics education. Journal for Research in Mathematics Education, 25(5), pp. 443-471.

KILPATRICK, J. (1994). Historia de la investigación en educación matemática, en Kilpatrick. J., Rico, L. y Sierra, M. Educación Matemática e Investigación, pp. 17-96 Madrid: Síntesis.

REYS, R. (2000). Doctorates in Mathematics Education: An Acute Shortage. Notice of the AMS, 47(10), pp. 1267-1270.

RICO, L. (1999a). Matemáticas, universidad y formación del profesorado. Revista Interuniversitaria de Formación del Profesorado, 34, pp. 245-262. poner en práctica los resultados de las propias investigaciones locales realizadas en educación matemática.

\section{NOTA}

* Esta investigación ha sido financiada por el V Programa propio de Fomento de la Investigación de la Universidad de Córdoba y el Plan Andaluz de Investigación de la Consejería de Educación y Ciencia de la Junta de Andalucía.
RICO, L. (1999b). Desarrollo en España de los estudios de doctorado en didáctica de la matemática, en Hart, K. y Hitt, F. (eds.). Dirección de tesis de doctorado en educación matemática: una perspectiva internacional, pp. 1-128. México: CINVESTAV.

RICO, L. y SIERRA, M. (2000). Didáctica de la matemática e investigación, en Carrillo, J. y Contreras, L.C. (eds.). Matemática española en los albores del siglo XXI. Huelva: Regué.

SCHOENFELD, A. H. (2000). Purposes and methods of research in mathematics education. Notice of the AMS, pp. 641-649.

SEIEM (2000). http://www,ugr,es/ seiem/Boletines/boletin1,htm, noviembre 12 de 2000.

TORRALBO, M., MAZ, A., RICO, L. y FERNÁNDEZ CANO, A. (2001). Programas de doctorado e investigación en didáctica de la matemática. Actas del Congreso Nacional de Didácticas Específicas. Las Didácticas de las Áreas Curriculares en el siglo XXI, vol. 1, pp. 905- 914. Granada: Grupo Editorial Universitario.

TORRALBO, M. (2001). «Análisis cientimétrico, conceptual y metodológico de las tesis doctorales españolas en educación matemática (1976-1998)». Tesis doctoral. Universidad de Granada.

TURUGET, D. (1989). Sociología de la ciencia: bibliometría, cientometría y cientografía. Noticias para Químicos, 316, pp. 4-12. 